\title{
NOON states in trapped ions
}

\author{
D. Rodríguez-Méndez and H.M. Moya-Cessa ${ }^{1}$ \\ ${ }^{1}$ INAOE, Coordinación de Optica, Apdo. Postal 51 y 216, 72000 Puebla, Pue., Mexico
}

\begin{abstract}
We show how NOON states may be generated in ion traps. We use the individual interaction of light with each of two vibrational modes of the ion to entangle them. This allows us to generate NOON states with $N=8$.
\end{abstract}

\section{INTRODUCTION}

Nonclassical states for mono-mode fields have attracted a great deal of attention over the years because of their fundamental and technological value, among them we have 1) macroscopic quantum superpositions of quasiclassical coherent states with different mean phases or amplitudes [1 [3], 2) squeezed states [4], and 3) the particularly important limit of extreme squeezing, i.e. Fock or number states 5]. More recently some bimodal fields have also attracted attention because they may be used for high precision phase measurements: NOON states, i.e., nonclassical states of combined photon pairs [6, 7]. Many applications in quantum imaging, quantum information and quantum metrology [8] depend on the availability of entangled photon pairs because entanglement is a distinctive feature of quantum mechanics that lies at the core of many new applications. These maximally path-entangled multiphoton states may be written in the form

$$
|N 00 N\rangle_{a, b}=\frac{1}{\sqrt{2}}\left(|N\rangle_{a}|0\rangle_{b}+|0\rangle_{a}|N\rangle_{b}\right)
$$

It has been pointed out that NOON states manifest unique coherence properties by showing that they exhibit a periodic transition between spatially bunched and antibunched states when undergo Bloch oscillations. The period of the bunching/antibunching oscillation is $\mathrm{N}$ times faster than the period of the oscillation of the photon density [9].

The greatest $N$ for which NOON states have been produced is $N=5[\underline{6}]$. Most schemes to generate this class of states are either for optical 6, 7] or microwave fields. In this contribution, we would like to analyze the possibility to generate them in ions [10 16], i.e. NOON states of their vibrational motion. We will show that they may be generated with $N=8$.

\section{ION-LASER INTERACTION}

The Hamiltonian for an ion in a two-dimensional Paul trap has the form

$$
\hat{H}=\nu_{x} a_{x}^{\dagger} a_{x}+\nu_{y} a_{y}^{\dagger} a_{y}+\frac{\omega_{e e}}{2} \hat{A}_{e e}+\left(\hat{A}_{g e} \lambda E^{(-)}(\hat{x}, \hat{y}, t)+H . c .\right),
$$

with $\lambda$ the electronic coupling matrix element, $E^{(-)}(\hat{x}, \hat{y}, t)$ the negative part of the classical electric field of the driving field. The operators $\hat{A}_{j k}$ take into account the transitions between the states $|j\rangle$ and $|k\rangle$ ( $g$ for ground and $e$ for excited). We assume the ion driven by a plane wave

$$
E^{(-)}(\hat{x}, \hat{y}, t)=E_{0} e^{-i\left(k_{x} \hat{x}+k_{y} \hat{y}+\omega t\right)}
$$

with $k_{j}, j=x, y$ the wavevectors of the driving field and define the Lamb-Dicke parameters

$$
\eta_{x}=2 \pi \frac{\sqrt{{ }_{x}\left\langle 0\left|\Delta \hat{x}^{2}\right| 0\right\rangle}}{\lambda_{x}}, \quad \eta_{y}=2 \pi \frac{\sqrt{{ }_{y}\left\langle 0\left|\Delta \hat{y}^{2}\right| 0\right\rangle_{y}}}{\lambda_{y}},
$$

such that we redefine

$$
k_{x} \hat{x}=\eta_{x}\left(a_{x}+a_{x}^{\dagger}\right), \quad k_{y} \hat{y}=\eta_{y}\left(a_{y}+a_{y}^{\dagger}\right) .
$$

In the resolved sideband limit, the vibrational frequencies $\nu_{x}$ and $\nu_{y}$ are much larger than other characteristic frequencies and the interaction of the ion with the two lasers can be treated separately, using a nonlinear Hamiltonian 
[17, 18]. The Hamiltonian (2) in the interaction picture can then be written as

$$
H_{I}=\left\{\begin{array}{lll}
\Omega_{x}^{(k)} e^{-\eta_{x}^{2} / 2} \hat{A}_{e g} \frac{\hat{n}_{x} !}{\left(\hat{n}_{x}+k\right) !} L_{\hat{n}_{x}}^{(k)}\left(\eta_{x}^{2}\right) a_{x}^{k}+H . c, & \eta_{y}=0, & \delta=k \nu_{x} \\
\Omega_{y}^{(k)} e^{-\eta_{y}^{2} / 2} \hat{A}_{e g} \frac{\hat{n}_{y} !}{\left(\hat{n}_{y}+k\right) !} L_{\hat{n}_{y}}^{(k)}\left(\eta_{y}^{2}\right) a_{y}^{k}+H . c, & \eta_{x}=0, & \delta=k \nu_{y}
\end{array}\right.
$$

where $L_{\hat{n}_{j}}^{(k)}, j=x, y$ are the operator-valued associated Laguerre polynomials, the $\Omega$ 's are the Rabi frequencies and $\hat{n}_{j}=a_{j}^{\dagger} a_{j}, j=x, y$. If we consider consider $\eta_{y}=0$ and $\eta_{x} \ll 1$ and take $\delta=4 \nu_{x}$ we obtain

$$
H_{I}^{(x)} \approx g A_{21} a_{x}^{4}+H . c .
$$

with $g=\Omega_{x} \frac{\eta_{x}^{4}}{4 !}$. The evolution operator for the interaction Hamiltonian is then (in the atomic basis, see [19])

$$
U_{I}^{(x)}(t)=e^{-i H_{I}^{(x)} t}=\left(\begin{array}{cc}
C_{\hat{n}}^{x} & -i S_{\hat{n}}^{\dagger x} V_{x}^{4} \\
-i V_{x}^{\dagger 4} S_{\hat{n}}^{x} & C_{\hat{n}-4}^{x}
\end{array}\right),
$$

where

$$
\begin{aligned}
C_{\hat{n}}^{x} & =\cos \left(\sqrt{\left(\hat{n}_{x}+4\right)\left(\hat{n}_{x}+3\right)\left(\hat{n}_{x}+2\right)\left(\hat{n}_{x}+1\right)} g t\right), \\
S_{\hat{n}}^{x} & =\sin \left(\sqrt{\left(\hat{n}_{x}+4\right)\left(\hat{n}_{x}+3\right)\left(\hat{n}_{x}+2\right)\left(\hat{n}_{x}+1\right)} g t\right),
\end{aligned}
$$

and the operator

$$
V_{x}=\frac{1}{\sqrt{\hat{n}_{x}+1}} a_{x}
$$

the Susskind-Glogower (phase) operator [20].

\section{GENERATION OF NOON STATES}

By starting with the ion in the excited state and the initial vibrational state in the vacuum state, i.e. $|0\rangle_{x}|0\rangle_{y}$, if we set $\eta_{y}=0$, after a convenient time, this is, the time when the probability to find the ion in its excited state is zero (meaning that the ion, by passing from its excited to its ground state, gives 4 phonons to the vibrational motion), we can generate the state $|4\rangle_{x}|0\rangle_{y}$. Repeating this procedure (with the ion reset again to the excited state, via a rotation), but now with $\eta_{x}=0$, for phonon are added to the $y$-vibrational motion, generating the two-dimensional state $|4\rangle_{x}|4\rangle_{y}$.

Therefore, if we consider the ion initially in a superposition of ground and excited states, and the $|4\rangle_{x}|4\rangle_{y}$ vibrational state

$$
\left|\psi_{\text {init }}\right\rangle=\frac{1}{\sqrt{2}}(|e\rangle+|g\rangle)|4\rangle_{x}|4\rangle_{y}
$$

for $\eta_{y}=0$ and $\tau_{p}$, the state generated is

$$
\left|\psi_{\eta_{y}=0}\right\rangle=\frac{i}{\sqrt{2}}\left(|e\rangle|0\rangle_{x}+|g\rangle|8\rangle_{x}\right)|4\rangle_{y} .
$$

Now, we consider this state as initial state for the next interaction with $\eta_{x}=0$ and still the interaction time $\tau_{p}$, to produce

$$
\left|\psi_{\eta_{x}=0}\right\rangle=-\frac{1}{\sqrt{2}}\left(|e\rangle|0\rangle_{x}|8\rangle_{y}+|g\rangle|8\rangle_{x}|0\rangle_{y}\right) .
$$

Next, the ion is rotated via a classical field (an on-resonance interaction) such that the state

$$
\left|\psi_{R}\right\rangle=-\frac{1}{2}\left[|e\rangle\left(|0\rangle_{x}|8\rangle_{y}-|8\rangle_{x}|0\rangle_{y}\right)+|g\rangle\left(|0\rangle_{x}|8\rangle_{y}+|8\rangle_{x}|0\rangle_{y}\right]\right.
$$


is obtained. Finally by measuring the ion in its excited state we produce the NOON state

$$
|N O O N\rangle_{e}=\frac{1}{\sqrt{2}}\left(|0\rangle_{x}|8\rangle_{y}-|8\rangle_{x}|0\rangle_{y}\right)
$$

and if the ion is measured in the ground state, also a NOON state is produced:

$$
|N O O N\rangle_{g}=\frac{1}{\sqrt{2}}\left(|0\rangle_{x}|8\rangle_{y}+|8\rangle_{x}|0\rangle_{y}\right),
$$

\section{CONCLUSIONS}

It has been shown a way to produce high NOON states $(N=8)$ by entangling the vibrational motion of an ion trapped in two dimensions. The entanglement is produced by a set of interactions of the trapped ion with laser fields conveniently tuned to produce 4-phonons transitions. It should be noted that, because the Lamb-Dicke regime is assumed, the four-phonon transitions we are proposing is difficult to achieve. Decoherence processes are usually not taken into account in ion-laser interactions because these interactions are, in general, not affected by the environment. However, due to the interaction times needed to generate the states presented here, they may be considered in this case. Here however we have only treated the ideal case as a first approach. We will study the consequences of the environment elsewhere.

[1] E. Schrödinger, Naturwissenschaften 23, 807; 823; 844 (1935).

[2] B. Yurke and D. Stoler, Phys. Rev. Lett. 57, 13 (1986).

[3] H. Moya-Cessa and A. Vidiella-Barranco, J. of Mod. Optics 42, 1547 (1995).

[4] R. Loudon and P.L. Knight, Special Issue of J. of Mod. Opt. 34, 709 (1987); H Moya-Cessa and A Vidiella-Barranco Journal of Modern Optics 39, 2481 (1992).

[5] J. Krause, M.O. Scully, T. Walther, and H. Walther, Phys. Rev. A 39, 1915 (1989).

[6] I. Afek, O.Ambar, and Y. Silberberg, Science 328, 879 (2010).

[7] J. P. Dowling, Contemp. Phys. 49, 125 (2008).

[8] F. Wolfgramm, A. Cere, and M. W. Mitchell, J. Opt. Soc. Am. B 27, A25(2010).

[9] Y. Bromberg, Y. Lahini, and Y. Silberberg, Phys. Rev. Lett. 105, 263604 (2011).

[10] D.J. Wineland, J.J. Bollinger, W.M. Itano, F.L. Moore, and D.J. Heinzen, Phys. Rev. A 46 R6797 (1992).

[11] D. Leibfried, R. Blatt, C. Monroe y D. Wineland, Rev. of Mod. Phys. 75281 (2003).

[12] J. Wineland et al., J. Res. Natl. Inst. Stand. Technol. 103259 (1998).

[13] D. Leibfried, D.M. Meekhof, B.E. King, C. Monroe, W.M. Itano, and D.J. Wineland, Phys. Rev. Lett. 77, 4281 (1996).

[14] S. Wallentowitz and W. Vogel, Phys. Rev. A 59, 531 (1999).

[15] M. Sasura and V. Buzek, J. of Mod. Optics 49, 1593 (2002).

[16] H. Moya-Cessa, D. Jonathan and P.L. Knight, J. of Mod. Optics 50, 265 (2003).

[17] R.L. de Matos Filho and W. Vogel, Phys. Rev. Lett. 76, 608 (1996).

[18] R.L. de Matos Filho and W. Vogel, Phys. Rev. A 54, 4560 (1996).

[19] S.J.D. Phoenix and P.L. Knight, J. of the Opt. Soc. Am B 7, 116 (1990).

[20] L. Susskind and J. Glogower, Physics 1, 49 (1964). 